\title{
Probing Nanoscale Structural Heterogeneity in Metallic Glasses Using 4-D STEM
}

Soohyun $\mathrm{Im}^{1}$, Zhen Chen ${ }^{2}$, Jared M. Johnson ${ }^{1}$, Pengyang Zhao ${ }^{1}$, Geun Hee Yoo ${ }^{3}$, Eun Soo Park ${ }^{3}$, Yunzhi Wang ${ }^{1}$, David A. Muller ${ }^{2}$, and Jinwoo Hwang ${ }^{1}$

1. Department of Materials Science and Engineering, The Ohio State University, Columbus, OH, USA

2. School of Applied and Engineering Physics, Cornell University, Ithaca, NY, USA

3. Department of Materials Science and Engineering, Seoul National University, Seoul, Korea

We present the direct determination of the heterogeneity at the nano to mesoscale structure of metallic glasses (MGs) using 4-D nanodiffraction in STEM. Shear localization is an important characteristic of MGs deformation that must be fully understood for further development of MGs and utilization of their superior properties [1]. However, unlike in crystalline materials where the structural defects responsible for plasticity are well defined, the structural origin of the shear deformation in MGs has remained elusive. The current understanding of MG deformation involves the concept of shear transformation zones (STZs), which are dynamic shear events that may occur within a group of a few tens to a few hundred atoms under stress [2]. The current knowledge gap in the field, however, is whether or how the STZ events are related to the local structure of the MG. The typical size of an STZ is proposed to be about one to a few nanometers, and therefore it matches the length scale of nanoscale medium range order (MRO) that constitutes the heterogeneity [3]. The match in the length scale suggests that STZs and local heterogeneity may be inherently coupled [4]. The potential relationship between MRO and STZ may provide important insights on, for example, why some MGs are much more ductile than others even by a small change in composition or thermal history. To study the relationship, we experimentally determine the heterogeneity in detail, including the type, size, distribution, and volume fraction of MRO, and investigate how it affects the shear localization and overall deformation of the MG [5].

The new generation of 4-D STEM detectors dramatically advances the experimental capability for this investigation (Fig. 1a and 1b). 4-D STEM provides the following important advantages over the conventional methods: (i) Due to the high frame rate, a large sample area can be examined with oversampling, which is important to directly determine the length scale of MRO. (ii) The rotational symmetry among the speckles in each diffraction pattern (Fig. 1c), which shows the type of the MRO, can be determined and directly related to the intensities of the dark field (DF) image constructed from the 4-D data. (iii) It is possible to form individual DF images as a function of the azimuthal angle, $\varphi$ (Fig. 1c, 1d, and 1e). Using these DF images, we can calculate the 2-D variance as a function of $\varphi$ over the entire sample area, $V_{x, y}(\varphi)$ (Fig. 1f), which can visualize the MRO regions much more reliably as compared to the images acquired using conventional HAADF-STEM or hollow-cone DF TEM.

The local volume that contains high degree of rotational symmetry can be identified as the MRO regions. We calculate the angular correlation function [6] to identify the rotational symmetry information (i.e. $n$-fold) for each nanodiffraction pattern, and the power spectrum amplitude of each symmetry is plotted in 2-D maps (Fig. 2a to 2f). The hotspot positions in each map mostly match the positions of the bright speckles in Fig. 1f, which are also shown on the 2-D map as the overlaid contour lines for comparison. This indicates that the speckles in $V_{x, y}(\varphi)$ image are indeed the diffraction from the MRO regions with high degree of local ordering. From these maps, we determine that the average size of the randomly distributed MRO is $\sim 1$ to $1.5 \mathrm{~nm}$, and the total volume fraction of MRO is $\sim 7.5 \%$ of the entire volume. This new information is very important, as it can be directly incorporated into the 
mesoscale deformation simulation (Fig. 2g) to reveal how the heterogeneity affects shear localization and overall deformation of MGs [7].

References:

[1] A.L. Greer, Y.Q. Cheng, and E. Ma, Materials Science and Engineering: R 74 (2013), p. 71.

[2] A.S. Argon, Acta Metallurgica,27(1) (1979), p. 47-58.

[3] J. Hwang et al., Physical Review Letters 108(19) (2012), p. 195505.

[4] J. Ding et al., Proceedings of the National Academy of Sciences 111 (2014), p. 14052.

[5] P. Zhao, J. Li, J. Hwang, and Y. Wang, Acta Materialia 134 (2017), p. 104.

[6] P. Wochner et al., Proceedings of the National Academy of Sciences 106 (2009), p. 11511.

[7] Work funded by NSF DMR-1709290, with 4D STEM at the PARADIM MIP (NSF DMR-1539918).

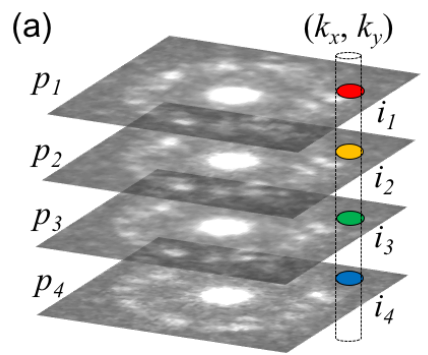

(b)

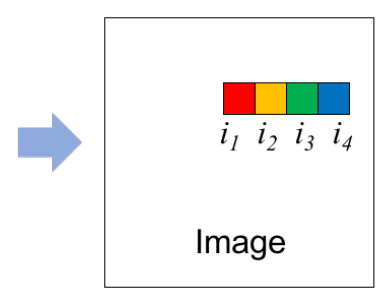

(c)

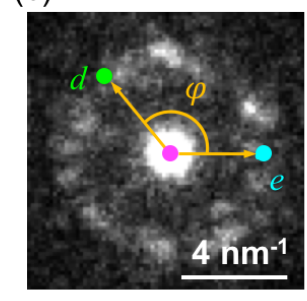

(d)

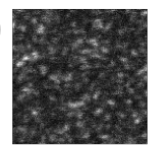

(e)

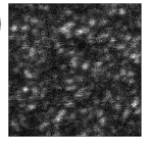

(f)

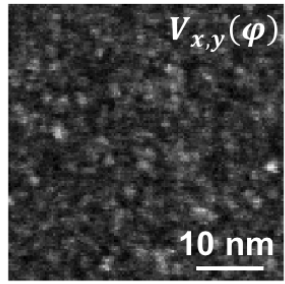

Figure 1. ( $a$ and $b$ ) A schematic showing 4-D STEM of $\mathrm{Zr}_{55} \mathrm{Co}_{25} \mathrm{Al}_{5} \mathrm{MG}$ using $1 \mathrm{~nm}$ probe. Any pixel in $k$-space can be selected to form the DF image in real space. DF images shown in (d) and (e) are formed using the $k$-space positions $d$ and $e$ shown in (c), respectively. (f) $V_{x, y}(\varphi)$ image showing MRO speckles.

(a) $\quad n=2$

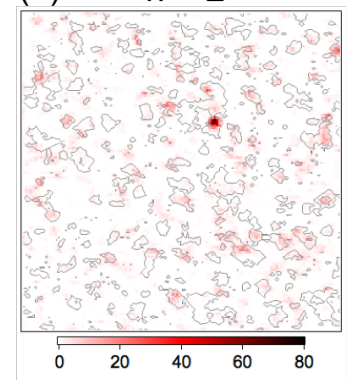

(d)

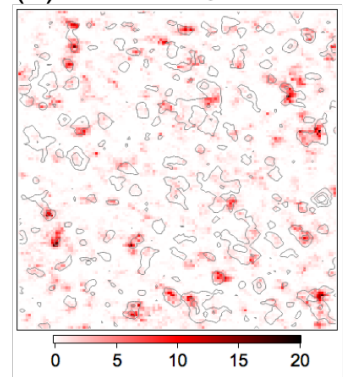

(b)

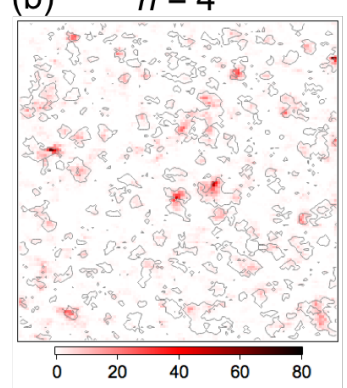

(e) $\quad n=10$

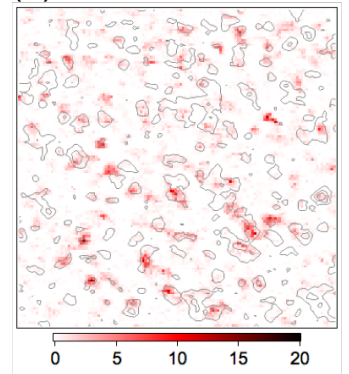

(c) $\quad n=6$

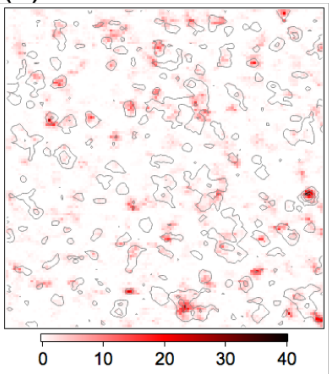

(f)

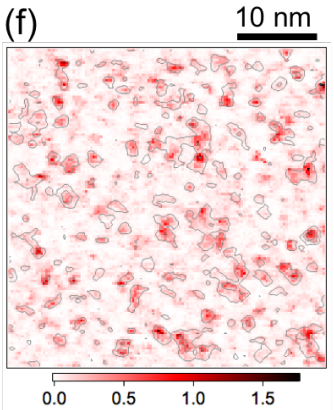

(g) $n=6,10 \%$
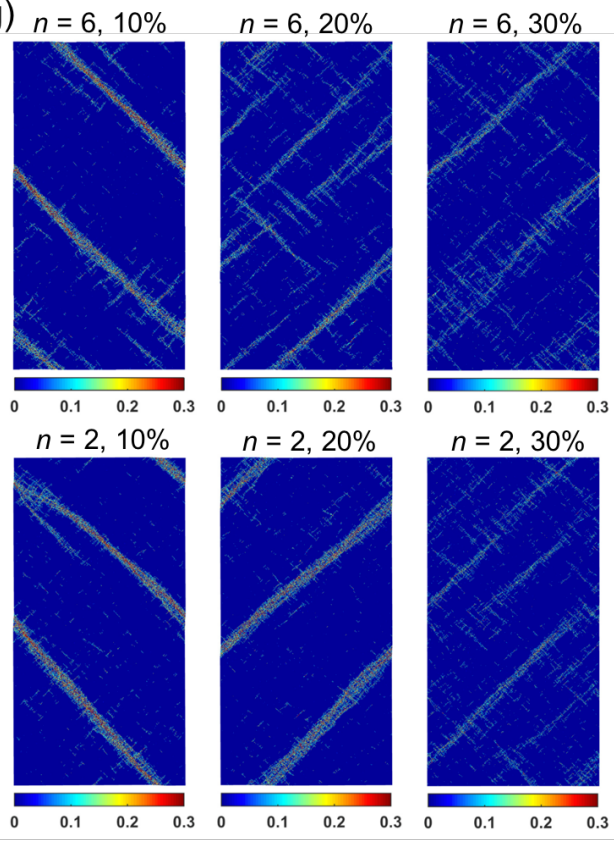
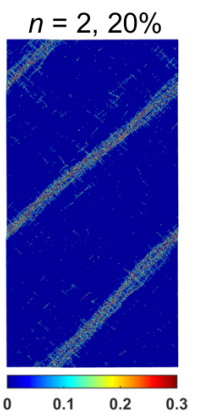
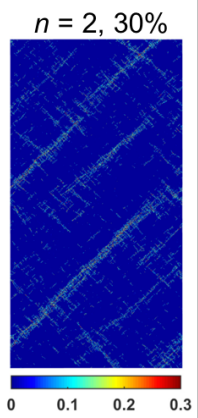

Figure 2. (a to e) Power spectrum maps showing the amplitude of the rotational symmetry for each $n$ value. The maps are also overlaid with the contour plots of the $V_{x, y}(\varphi)$ shown in Fig. 1f for comparison. (f) is the normalized sum of all the maps in (a) to (e). (g) Mesoscale kinetic Monte Carlo deformation simulation of an MG incorporating the experimentally determined heterogeneity, $n=2$ and $n=6$ [5]. 